\title{
GRANULITOS DA FAIXA PARAÍBA DO SUL: CARACTERES QUIMMICOS DOS PIROXÊNIOS E VALORES GEOTERMOMÉTRICOS
}

\author{
MARCOS AURÉLIO FARIAS DE OLIVEIRA*
}

\begin{abstract}
Seven pairs of clino and orthopyroxenes from granulites belonging to the Paraíba do Sul belt (Atlantic granulite belt) have been chemically analysed by electron microprobe method.

Thermodynamic equilibrium among coexisting pyroxenes is evident from the textural patterns; distribution coefficient for Fe-Mg and the crystallization trends. Geothermometric calculations indicate temperatures of about $850^{\circ} \mathrm{C}$ for the equilibration of the minerals. These values point to the formation of the Paraiba do Sul pyroxenes under high grade metamorphic conditions.
\end{abstract}

INTRODUÇÃo A faixa Paraiba do Sul, parte integrante do Cinturão Granulítico Attântico (Fyfe e Leonardos Jr., 1974; Wernick e Almeida, 1979), é constituída por um conjunto de rochas que incluem migmatitos diversos, gnaisses e granulitos com intercalaçðes menores de quartzitos, mármores e rochas calcossilicáticas. Entre essas rochas destacam-se charnockitos e granulitos mais ou menos cataclásticos, com estruturas bandadas e fitadas, às vezes blastomiloníticas e que localmente assumem aspecto migmatítico com as porçżes mais ácidas, charnockíticas, envolvendo porçðes mais básicas de composição enderbítica até gábrica.

No presente trabalho são apresentados resultados referentes ao estudo desses granulitos em relação à presença de orto e clinopiroxênios que constituem elementos essenciais de muitas dessas rochas. Pormenores geológicos sobre a região bem como sobre o quimismo das litologias de alto grau metamórfico ora mencionadas podem ser encontrados em Oliveira (1980) e Oliveira (1982, em preparação).

Orto e clinopiroxênios ocorrem freqüentemente juntos e em equilibrio, principalmente em tipos enderbíticos e noríticos, tendo sido registrados também em alguns tipos mais ácidos. As fortes deformações com recristalizaçð̃es que transformaram essas rochas em blastomilonitos não foram suficientes, na maioria das amostras, para destrui-los e nem mesmo o equilibrio entre eles, que é herança de ciclo anterior. Assim, o que se observa nas lâminas petrográficas são os restos corroídos de orto e clinopiroxênios, sem qualquer evidência de recristalização posterior de fases semelhantes. Apenas biotita e hornblenda aparecem como produtos de eventos metamórficos mais recentes.

Entre as numerosas ocorrências de rochas que apresentam piroxênios em toda a área, foram selecionadas quinze amostras, das quais, por motivos técnicos, só sete tiveram suas análises completas. Pela Fig. 1 pode-se ver que elas possuem ampla distribuição, estendendo-se de Três Rios a Carangola e, apesar de estarem muito distantes uma das outras, são petrograficamente muito semelhantes.

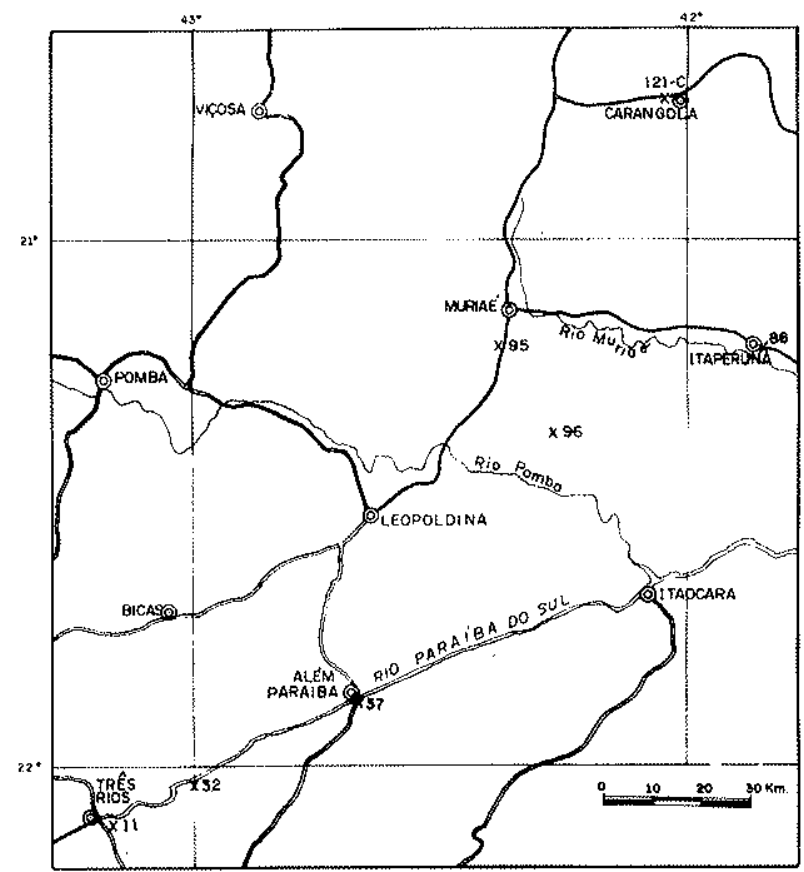

Figura I - Localizaçao das amostras estudadas

DADOS Químicos A composição química dos orto e clinopiroxênios foi obtida utilizando-se uma microssonda eletrônica marca Jeol, equipada com dois espectrômetros, do Laboratório de Química Industrial da Université Libre de Bruxelas. As condições instrumentais foram as seguintes: potencial de aceleração, $15 \mathrm{kV}$; corrente da amostra, $0,08 \mu \mathrm{A}$; tempo de integração $10 \mathrm{~s}$; e diâmetro do feixe eletrônico, aproximadamente $1 \mu$. Os padrōes utilizados foram oxidos puros dos elementos analisados. Foram feitas correções para absorção, número atômico e efeitos de matriz, utilizando um programa baseado no método de Philibert (1964). Não se observou nenhum zo-

* Instituto de Geociências e Ciências Exatas, Universidade Estadual Paulista (UNESP), Campus de Rio Claro - Rio Claro, SP 
Tabela l - Análises químicas dos ortopiroxênios

\begin{tabular}{c|c|c|c|c|c|c|c}
\hline & 96 & $121-\mathrm{C}$ & 37 & 32 & 88 & 11 & 95 \\
\hline $\mathrm{SiO}_{2}$ & 51,67 & 53,34 & 51,46 & 49,82 & 51,95 & 50,73 & 52,73 \\
\hline $\mathrm{TiO}_{2}$ & 0,08 & 0,10 & 0,03 & 0,08 & 0,10 & 0,07 & 0,06 \\
\hline $\mathrm{Al}_{2} \mathrm{O}_{3}$ & 1,00 & 0,65 & 1,14 & 0,55 & 1,72 & 0,44 & 0,89 \\
\hline $\mathrm{FeO}$ & 32,56 & 23,76 & 27,73 & 33,47 & 26,20 & 34,85 & 27,34 \\
\hline $\mathrm{MnO}$ & 0,50 & 0,54 & 0,76 & 0,78 & 0,70 & 0,82 & 1,10 \\
\hline $\mathrm{MgO}$ & 15,35 & 22,36 & 18,72 & 13,82 & 18,91 & 13,44 & 18,17 \\
\hline $\mathrm{CaO}$ & 0,71 & 0,57 & 0,50 & 0,58 & 0,51 & 0,56 & 0,19 \\
\hline Total & $\mathbf{1 0 1 , 8 6}$ & $\mathbf{1 0 1 , 3 2}$ & $\mathbf{1 0 0 , 3 4}$ & $\mathbf{9 9 , 1 0}$ & $\mathbf{1 0 0 , 0 9}$ & $\mathbf{1 0 0 , 9 1}$ & $\mathbf{1 0 0 , 4 8}$ \\
\hline
\end{tabular}

Número de ions na base de seis oxigênios

\begin{tabular}{l|c|c|c|c|c|c|c}
\hline $\mathrm{Si}$ & 1,981 & 1,973 & 1,963 & 1,985 & 1,969 & 1,992 & 2,000 \\
\hline $\mathrm{A} l^{\mathrm{iv}}$ & 0,019 & 0,027 & 0,037 & 0,015 & 0,031 & 0,008 & - \\
\hline $\mathrm{Al} \mathrm{I}^{\mathrm{vi}}$ & 0,026 & 0,001 & 0,014 & 0,011 & 0,046 & 0,012 & 0,040 \\
\hline $\mathrm{Ti}$ & 0,002 & 0,003 & 0,001 & 0,002 & 0,003 & 0,002 & 0,002 \\
\hline $\mathrm{Fe}$ & 1,044 & 0,735 & 0,885 & 1,115 & 0,830 & 1,144 & 0,868 \\
\hline $\mathrm{Mn}$ & 0,016 & 0,017 & 0,024 & 0,026 & 0,022 & 0,027 & 0,035 \\
\hline $\mathrm{Mg}$ & 0,877 & 1,233 & 1,065 & 0,821 & 1,068 & 0,787 & 1,027 \\
\hline $\mathrm{Ca}$ & 0,029 & 0,022 & 0,020 & 0,025 & 0,021 & 0,024 & 0,008 \\
\hline $\mathrm{Z}$ & 2,000 & 2,000 & 2,000 & 2,000 & 2,000 & 2,000 & 2,000 \\
\hline $\mathrm{WX}$ & 1,994 & 2,011 & 2,009 & 2,000 & 1,990 & 1,996 & 1,980 \\
\hline $\mathrm{Ca}$ & 1,47 & 1,10 & 1,00 & 1,26 & 1,08 & 1,21 & 0,41 \\
\hline $\mathrm{Mg}$ & 44,61 & 61,43 & 53,41 & 41,32 & 55,03 & 39,71 & 52,99 \\
\hline $\mathrm{Fe}+\mathrm{Mn}$ & 53,92 & 37,47 & 45,59 & 57,42 & 43,89 & 59,08 & 46,60 \\
\hline $\mathrm{Fe} / \mathrm{Mg}$ & 1,19 & 0,60 & 0,83 & 1,36 & 0,78 & 1,45 & 0,84 \\
\hline $\mathrm{T}^{\circ} \mathrm{C}^{*}$ & 812 & 874 & 843 & 834 & 888 & 819 & 839 \\
\hline $\mathrm{T}^{\circ} \mathrm{C}^{* *}$ & 861 & 902 & 887 & 900 & 950 & 826 & 882 \\
\hline $100 \mathrm{Mg}$ & $\mathbf{4 5 , 3}$ & $\mathbf{6 2 , 1}$ & $\mathbf{5 4 , 0}$ & $\mathbf{4 1 , 8}$ & $\mathbf{5 5 , 6}$ & $\mathbf{4 0 , 2}$ & $\mathbf{5 3 , 2}$ \\
\hline $\mathrm{Mg}+\mathrm{Fe}+\mathrm{Mn}$ & & & & & & \\
\hline
\end{tabular}

(1) Todo ferro calculado como FeO

* Wood e Banno (1973)

** Wells (1976)

neamento nos minerais analisados, tendo sido registrados cerca de 30 pontos por grão investigado, com os resultados finais dados pela média dessas leituras.

$O$ resultado dessas análises é apresentado nas Tabs. 1 e 2, respectivamente, para ortopiróxênios e clinopiroxênios. Constam também dessas tabelas a fórmula química, alguns índices de diferenciação, os valores do coeficiente de distribuição $\mathrm{Fe} \cdot \mathrm{Mg}$, conforme Davidson (1968), e as temperaturas calculadas com base nas calibragens de Wood e Banno (1973) e Wells (1977).

Para as análises na microssonda foram selecionados grãos de orto e clinopiroxênios que estivessem em contato, garantindo assim o equilibrio entre ambos. Os sete ortopiroxênios analisados exibem como características mais marcantes baixos teores de $\mathrm{CaO}(0,19$ a 0,71$)$, teores de $\mathrm{Al}_{2} \mathrm{O}_{3}$ entre 0,44 e 1,72, e quantidades da moléçla enstatita situadas entre 40 e 64 , correspondendo principalmente ao termo hiperstênio da série.

Já os clinopiroxênios apresentam como feições marcantes um teor quase constante de $\mathrm{CaO}$ em torno de $22 \%$ e variações maiores nos teores de $\mathrm{MgO}$ e FeO e $\mathrm{Al}_{2} \mathrm{O}_{3}$. Os termos representados são a salita e a ferrossalita da série diopsídio hedenbergita (Deer et al., 1963).
Nas Figs. 2 e 3, as amostras analisadas foram projetadas no quadrilátero diopsidio (Di)-hedenbergita (Hd)-ferrossilita(Fs)-enstatita (En) e observa-se que os pares de orto e clinopiroxênio, que coexistem em equilíbrio, evoluem de maneira semelhante, verificando-se um enriquecimento em ferro. Os clinopiroxênios mostram pequena queda nos teores de $\mathrm{CaO}$, o que não se verifica para os ortopiroxênios. As tendências obtidas para as rochas granuliticas da faixa Paraíba do Sul (Fig. 3) coincidem aproximadamente com as tendências metamórficas de Madras, Índia (Howie, 1955), Broken-Hill-Quairading (Davidson, 1968), além de outras como Pien (Girardi, 1974) e São José do Rio Pardo (Oliveira e Hipólito, 1978). Afastam-se, porém, da tendência das rochas magmáticas de Skaergaard (Brown, 1957; e Brown e Vincent, 1963).

$O$ estado de equilibrio termodinâmico entre os dois minerais, já evidenciado na Fig. 2, é novamente confirmado na Fig. 4, onde foram plotados os valores de $\mathrm{Al}_{2} \mathrm{O}_{3}$ dos pares de piroxênios. Ficam evidentes os baixos teores desse óxido quando comparados aos terrenos granulíticos da Austrália (Wilson, 1976), destacando-se porém a relação linear obtida com a projeção dos valores de ambos os piroxênios. 
Tabela 2 - Análises químicas dos clinopiroxênios

\begin{tabular}{l|c|c|c|c|r|r|r}
\hline & 96 & 88 & 32 & $121 \cdot \mathrm{C}$ & 37 & 11 & 95 \\
\hline $\mathrm{SiO}_{2}$ & 52,25 & 53,31 & 52,86 & 52,48 & 52,04 & 52,06 & 62,93 \\
\hline $\mathrm{TiO}_{2}$ & 0,11 & 0,17 & 0,11 & 0,13 & 0,17 & 0,11 & 0,11 \\
\hline $\mathrm{Al}_{2} \mathrm{O}_{3}$ & 1,45 & 2,42 & 1,03 & 1,19 & 1,38 & 1,02 & 1,43 \\
\hline $\mathrm{FeO}^{*}$ & 13,57 & 9,67 & 13,23 & 7,68 & 10,98 & 15,15 & 9,52 \\
\hline $\mathrm{MnO}$ & 0,18 & 0,24 & 0,30 & 0,20 & 0,30 & 0,34 & 0,40 \\
\hline $\mathrm{MgO}$ & 11,05 & 13,39 & 11,38 & 14,33 & 13,45 & 10,52 & 12,89 \\
\hline $\mathrm{CaO}$ & 22,23 & 21,92 & 21,84 & 22,14 & 22,35 & 21,68 & 22,44 \\
\hline Total & $\mathbf{1 0 0 , 8 4}$ & $\mathbf{1 0 1 , 1 2}$ & $\mathbf{1 0 0 , 7 5}$ & $\mathbf{9 8 , 1 5}$ & $\mathbf{1 0 0 , 6 7}$ & $\mathbf{1 0 0 , 8 8}$ & $\mathbf{9 9 , 7 2}$ \\
\hline
\end{tabular}

Número de íons na base de seis oxigênios

\begin{tabular}{|c|c|c|c|c|c|c|c|}
\hline$\underline{\mathrm{Si}}$ & 1,972 & 1,964 & 1,990 & 1,981 & 1,949 & 1,971 & 1,984 \\
\hline $\mathrm{Al}^{\mathrm{iv}}$ & 0,028 & 0,036 & 0,010 & 0,019 & 0,051 & 0,029 & 0,016 \\
\hline $\mathrm{Al}^{\mathrm{Vi}}$ & 0,036 & 0,069 & 0,036 & 0,034 & 0,010 & 0,016 & 0,047 \\
\hline$\underline{\mathrm{Ti}}$ & 0,003 & 0,005 & 0,003 & 0,004 & 0,005 & 0,003 & 0,003 \\
\hline $\mathrm{Fe}$ & 0,428 & 0,298 & 0,416 & 0,242 & 0,344 & 0,480 & 0,298 \\
\hline $\mathrm{Mn}$ & 0,005 & 0,007 & 0,10 & 0,006 & 0,009 & 0,011 & 0,013 \\
\hline $\mathrm{Mg}$ & 0,621 & 0,735 & 0,639 & 0,806 & 0,750 & 0,594 & 0,720 \\
\hline $\mathrm{Ca}$ & 0,899 & 0,865 & 0,881 & 0,895 & 0,897 & 0,880 & 0,901 \\
\hline $\mathrm{Z}$ & 2,000 & 2,000 & 2,000 & 2,000 & 2,000 & 2,000 & 2,000 \\
\hline WXY & 1,992 & 1,979 & 1,985 & 1,987 & 2,015 & 1,984 & 1,982 \\
\hline $\mathrm{Ca}$ & 46,03 & 45,41 & 45,27 & 45,92 & 44,85 & 44,78 & 46,63 \\
\hline $\mathrm{Mg}$ & 31,80 & 38,58 & 32,84 & 41,36 & 37,50 & 30,23 & 37,27 \\
\hline $\mathrm{Fe}+\mathrm{Mn}$ & 22,17 & 16,01 & 21,89 & 12,72 & 17,65 & 24,99 & 16,10 \\
\hline $\mathrm{Fe} / \mathrm{Mg}$ & 0,69 & 0,40 & 0,65 & 0,30 & 0,46 & 0,81 & 0,41 \\
\hline$K_{D}{ }^{* *}$ & 1,72 & 1,95 & 2,09 & 2,00 & 1,80 & 1,79 & 2,04 \\
\hline
\end{tabular}

* Todo ferro calculado como FeO

${ }^{* *} K_{D}(o p x-c p x)=(\mathrm{Fe} / \mathrm{Mg}) o p x /(\mathrm{Fe} / \mathrm{Mg}) c p x$

COEFICIENTES DE DISTRIBUIÇÃO Fe-Mg .. Os coeficientes de distribuição dos elementos $\mathrm{Fe}$ e $\mathrm{Mg}$ para os pares de piroxênios dos granulitos da faixa Paraíba do Sul foram calculados segundo a fórmula $K_{D}(\mathrm{Fe})$ opx $c p x$ $=(\mathrm{Fe} / \mathrm{Mg}) o p x .(\mathrm{Fe} / \mathrm{Mg}) c p x$, conforme indicação de Davidson (1968). Como as análises são obtidas por microssonda e não há possibilidade de se separarem os teores de $\mathrm{Fe}^{2+}$ e $\mathrm{Fe}^{3+}$, os cálculos do $K_{D}$ foram feitos colocandose na fórmula o ferro total, o que provoca nos resultados finais diferenças inferiores a $10 \%$, em geral para menos. Os valores médios obtidos para o $K_{D}$ situam-se em torno de 1,9 e são comparáveis aos de outras áreas metamórficas de catazona, como as da Província de Greenville no Canadá (Nantel, 1977) e as de Fraser Range na Austrália ocidental (Wilson, 1976).

$\mathrm{Na}$ Fig. 5, foram plotadas as razões $\mathrm{Fe} / \mathrm{Mg}$ dos orto e clinopiroxênios, evidenciando uma distribuição aproximadamente retilínea dos pontos $\left(K_{D}\right)$, o que diz novamente a favor do equilíbrio entre os pares analisados.

GEOTERMOMETRIA Foi Kretz (1963) quem inicialmente tentou estabelecer uma relação entre a repartição $\mathrm{Fe}-\mathrm{Mg}$ dos pares de piroxênios naturais em equilíbrio e a temperatura para as rochas magmáticas e metamórficas. A partir daí, vários trabalhos desenvolveram-se neste sentido, destacando-se principalmente os de Wood e Banno (1973). O geotermômetro idealizado por esses autores ba-

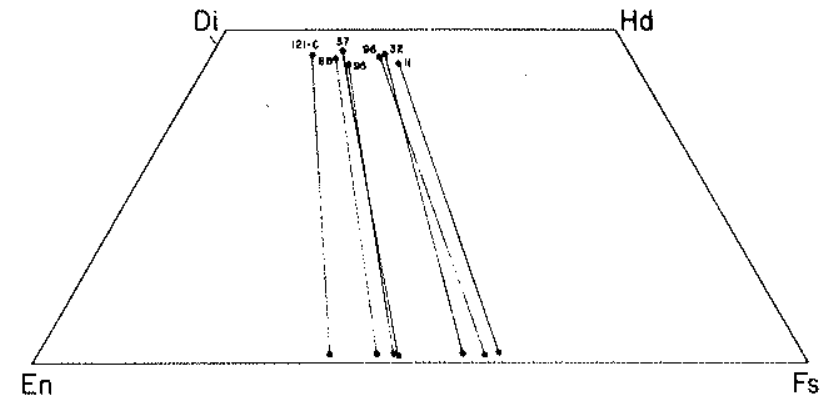

Figura 2 - Composição química de clino e ortopiroxênios coe xistentes projetadas no tetraedro Di-Hd-En-Fs

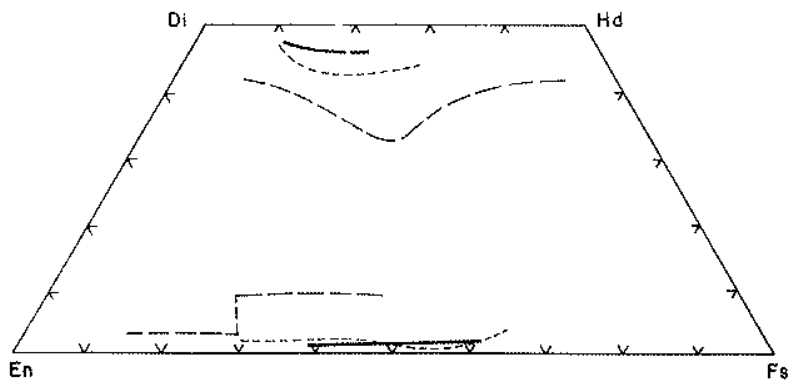

Figura 3 - Tendências de cristalização dos piroxênios da faixa granulitica Paraiba do Sul (linha contínua) comparadas às tendências da provincia metamórfica de Madras (Howie, 1955 - (raços curtos) e da intrusão de Skaergaard (Brown, 1957; e Brown e Vincent, 1963 - traços longos) 


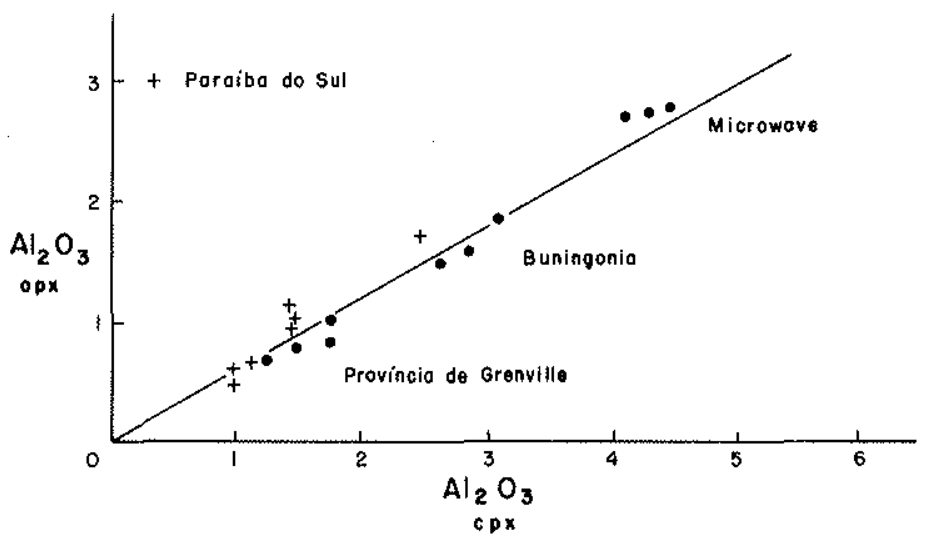

Figura 4 - Relaçao $\mathrm{Al}_{2} \mathrm{O}_{3}$ opx- $\mathrm{Al}_{2} \mathrm{O}_{3} \mathrm{cpx}$ para os pares de Wilson (1976), Nantel (1977) e do Paralba do Sul

seia-se na complexa distribuição intracristalina dos íons $\mathrm{Fe}^{2+}$ e $\mathrm{Mg}^{2+}$ em quatro posiçōes estruturais do orto e clinopiroxênio, e no conceito de atividades. A proposição de Wood e Banno (1973) foi posta em questão por Wells (1977), que sugere pequenas modificações em função dos altos teores de Fe dos piroxênios em equilíbrio, que teriam influência no valor do $K_{D}$ desses minerais. Segundo demonstra em seu trabalho, a pressões e temperaturas constantes, a concentração de $\mathrm{Ca}$ diminui nos clinopiroxênios com o aumento dos teores de Fe.

A aplicação do geotermômetro de Wood e Banno (1973) para os piroxênios granulitos da faixa Paraíba do Sul forneceu resultados que variam de $812^{\circ} \mathrm{C}$ a $888^{\circ} \mathrm{C}$, com valor médio de $844^{\circ} \mathrm{C}$ (Tab. 1), perfeitamente compatíveis com áreas de catazona e comparáveis com outras áreas semelhantes do mundo (Nantel, 1977). Segundo Hewis (1975), a precisão da calibragem de Wood e Banno (1973) está em torno de $50^{\circ} \mathrm{C}$.

$\mathrm{Na}$ Tab, 1 são apresentados também os valores de temperatura obtidos pela calibragem de Wells (1977). Os resultados são um pouco superiores aos primeiros e situam-se entre $826^{\circ} \mathrm{C}$ e $950^{\circ} \mathrm{C}$, com a média de $887^{\circ} \mathrm{C}$. Observa-se maior dispersão dos dados, sendo a diferença entre as temperaturas máxima e mínima superior a $100^{\circ} \mathrm{C}$, fato que não deve ser esperado, pois, apesar da grande distância que separa as amostras investigadas, as paragêneses minerais são muito semelhantes em toda a área. De qualquer modo, os resultados médios obtidos não se afastam muito dos calculados pelo geotermômetro de Wood e Banno (1973).

Quanto às pressōes de formação dessas rochas, a julgar pelas indicações da Fig. 4, admite-se que não tenham sido muito altas e se situem em torno de $6-7 \mathrm{~kb}$. Essa estimativa baseia-se nos dados de Wilson (1976), mostrando que os piroxênios formados a pressðes mais elevadas contêm teores maiores de $\mathrm{Al}_{2} \mathrm{O}_{3}$, fato exemplificado pelos granulitos de Microwave na Austrália.

Como conclusão, pode-se dizer que os valores ora obtidos se coadunam perfeitamente com a classificaçăo dessas rochas dentro da fácies granulito e permitem supor que, durante o metamorfismo, as reaçoes de "charnockitização" levaram ao aparecimento de líquidos ricos em álcalis que seriam retirados do sistema, provocando recristalização de tipos petrográficos mais "secos" e empobrecidos nesses elementos. Os valores de $T$ foram sufi-

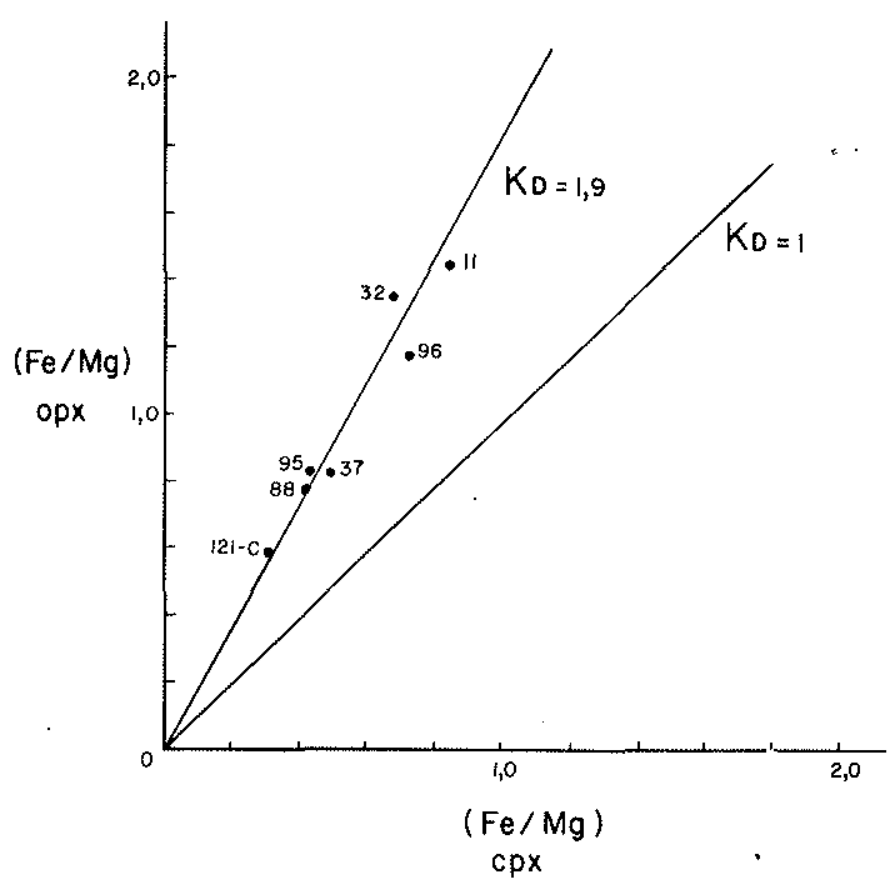

Figura 5 - Diagrama $\mathrm{Fe} / \mathrm{Mg}$ opx-Fe/Mg $\mathrm{cpx}, \mathrm{K}_{\mathrm{D}}=1,9$

cientemente altos para provocar anatéxis mesmo nas faces menos hidratadas, gerando migmatitos "secos", onde mobilizados charnockíticos envolvem tipos mais básicos, enderbíticos. Além disso, as condiçðes de pressão e temperatura atingidas durante o processo metamórfico, que culminou com a formação dos granulitos, permitem estimar em $30 \mathrm{~km}$ a profundidade em que tal processo ocorre, inferindo-se assim um gradiente geotérmico entre $30^{\circ} \mathrm{C}$ e $35^{\circ} \mathrm{C} / \mathrm{km}$.

Agradecimentos Registramos aqui nossos agradecimentos ao Conselho Nacional de Desenvolvimento Científico e Tecnológico $(\mathrm{CNPq})$ pelo suporte financeiro. Agradecemos também a M. Naessens do Laboratório de Química Industrial da Université Libre de Bruxelas, que nos permitiu a utilização da microssonda, e aos Drs. Jean Michot e Jacques Delhal, com quem mantivemos discussð̃es proveitosas. 


\section{BIBLIOGRAFIA}

BROWN, G.M. - 1957 - Pyroxenes from the early and middle stages of fractionation of the Skaergaard intrusion, East Greenland. Mon. May. 31: 511-543. BROWN, G.M. e VINCENT, E.A. - 1963 - Pyroxenes from the late stage of fractionation of the Skaergaard intrusion, East Greenland. J: Petrol. 4: $175-196$.

DAVIDSON, L.R. - 1968 - Variation in ferrous iron-magnesium distribution coefficients of metamorfphic pyroxenes from Quairading, Western Australia. Contr. Miner. Petrol. 19: 239-259.

DEER, W.A., HOWIE, R.A. e ZUSSMAN, J. - 1963 - Rock Forming Minerals, vol. II, Chain silicates, Lonymans, Londres.

FYFE, W.S. e LEONARDOS Jr., O.H. - 1974 - Ancient Metamorphic - Migmatite Belts of the Brazilian Atlantic Coast: The African Connection. Rev. Bras. Geoc., $4: 274-251$.

GIRARDI, V.A.V. - 1974 - Petrologia do Complexo Básico-ultrabásico de Pien, PR. Tese de Livre-Docência, Instituto de Geociências, Universidade de São Paulo (inédito). •

HEWINS, R.H. - 1975 - Pyroxene geothermometry of some granulite facies rocks. Contr. Mineral. Petrol. 50: 205-209.

HOWIE, R.A. - 1955 - The geochemistry of the charnockites series of Madras, India. Tran. Royal Soc. Edinburgh, 62: 725-769.

KRETZ, R - 1963 - Distribution of magnesian and iron between orthopyroxenes and calcic pyroxenes in natural assemblages. J. Geol. 7: 773-785.

NANTEL, S. - 1977 - Le Problème des Equilibres Cordierite-grenat et Orthopyroxène-clynopyroxene en Catazone. Application à la Géothermobarométrie dans le Sud de la Province de Greenville. Tese de Mestrado. Departamento de Geologia, Universidade de Montreal (inédito).
OLIVEIRA, M.A.F. de e HYPÓLITO, R. - 1978 - Ortopiroxênios e clinopiroxênios coexistentes nos granulitos de Săo José do Rio Pardo, SP. Rev. Bras. Geoc. 8: 249-261

OLIVEIRA, M.A.F. de - 1980 - Petrologia das Rochas Granulíticas da Faixa Paraiba do Sul, Estados do Rio de Janeiro e Minas Gerais. Tese de LivreDocência. Instituto de Geociências e Ciências Exatas, UNESP (inédito).

OLIVEIRA, M.A.F. de - 1982 - Granulitos da fajxa Paraiba do Sul: Petrografia e quimismo. Em preparaçåo.

PHILIBERT, J, - 1964 - L'analyse quantitative en micro-analyses par sonde életronique. Métaux Corrosion Industries, 465

WELLS, P.R.A - 1977 - Pyroxene thermometry in simple and complex systems. Contr. Mineral. Petrol. 62: 129-140.

WERNICK, E. e ALMEIDA, F.F.M. de - 1979 - The geotectonic environment of early Precambrian granulites in Brazil. Precambrian Research, 8: 1-17.

WILSON, A.F - 1976 - Aluminium in coexisting pyroxenes as a sensitive indicator of changes in metamorphic grade within the mafic granulite terrane of the fraser Range, Western Australia. Contr. Miner. Petrol. 56: 255-277.

WOOD, B.J. \& BANNO, S. - 1973 - Garnet-orthopyroxene and orthopyroxeneclinopyroxene relationships in simple and complex systems. Contr. Mineral. Petrol. 42: 109-124. 